\title{
A Closer Look at the Visual Input to Self-Motion Estimation
}

John A. Perrone

Psychology Department, The University of Waikato, Hamilton, New Zealand

\section{Introduction}

In their Chapter, Dahmen et al. use an interesting approach to the self-motion (egomotion) estimation problem that combines traditional vector flow field decomposition schemes with a matched filter (template) scheme. In the field of visual self-motion perception these two approaches represent two different schools of thought about how biological systems solve the self-motion problem. The boundaries between these classes of models occasionally become blurred but there is one feature that distinguishes these two approaches and which can be used to assess their pros and cons: namely the type of input they are designed to process. In this section I examine the different input requirements of the two model types and identify some of the challenges that are faced by modellers in this area.

It has long been suggested that visual navigation through the environment relies on our ability to detect and respond to specific patterns of retinal velocity or optic flow (Gibson 1950). There is ample empirical evidence that the self-motion problem can be solved using just visual motion information (e.g., Rieger and Toet 1985; Cutting 1986; Warren and Hannon 1990; Stone and Perrone 1997). It is also well established that - in theory - the instantaneous retinal optic flow contains sufficient information for the recovery of information about our instantaneous retino-centric heading (3D translation direction), eye-body rotation, and the relative depth of points in the world (Koenderink and van Doorn 1975; LonguetHiggins and Prazdny 1980; Zacharias et al. 1985; Heeger and Jepson 1992). These analyses demonstrated that the equations governing the projection of an environmental point $(\mathrm{x}, \mathrm{y}, \mathrm{z})$ onto an image plane during movement of an observer, could be solved for the observer motion parameters, given a small number of image velocity vectors. Some of these mathematical considerations motivated models of human visual self-motion estimation. 


\section{Models of human visual self-motion estimation}

\subsection{Vector-based decomposition models}

Most current models of self-motion estimation are inherently vector based (e.g., Koenderink and van Doorn 1975; Rieger and Lawton 1985; Heeger and Jepson 1992; Hildreth 1992; Lappe and Rauschecker 1993; Dyre and Andersen 1994; Royden 1997; Dahmen et al., this volume). Their algorithms assume that local velocity vectors have been derived from the retinal input imagery. At some stage, the input is assumed to be a distribution of velocity vectors $(\mathbf{x}, \mathbf{y})$ and the algorithms perform some calculation on these vectors (e.g., vector subtraction). The impetus for using the vectors is that the theoretical analyses of the flow fields have turned up some clever schemes that enable the local flow vectors arising from combined translation and rotation of the observer to be "decomposed" into a translation component and a rotation component. For example, by subtracting one vector from another in the same small local region, only the translation component remains because the rotation component is common to both vectors (LonguetHiggins and Prazdny 1980; Rieger and Lawton 1985). This is very appealing because the observer heading direction can then - in theory - be derived from the vector flow field translation components even though the observer was rotating during the translation.

\subsection{Template models}

For primates at least, the current evidence casts some doubts on the suggestion that the clever but often complex algorithms underlying the decomposition schemes are used to estimate self-motion. Neurones in the Medial Superior Temporal area (MST) that have been identified as having a role in self-motion estimation (e.g., Saito et al. 1986; Tanaka et al. 1986; Duffy and Wurtz 1991a, b) do not display the critical properties one would expect from a system based on decomposition of the flow field (Perrone and Stone 1998). Their receptive field properties are more consistent with schemes that simply register the global patterns of image motion directly (e.g., Perrone 1992; Perrone and Stone 1994) without resorting to vector subtraction or other forms of vector algebra. Such a "template" based approach to the self-motion estimation problem was proposed as an alternative to decomposition models by a number of people. In the insect vision area, a number of large field motion sensitive neurones had been postulated as being involved in visual course stabilization and gaze control (Hengstenberg 1982; Hausen 1993). In primates, a group of Japanese researchers (Saito et al. 1986; Tanaka et al. 1986; Tanaka and Saito 1989) suggested that MT (Middle Temporal) neurones could be assembled into networks that responded best to the radial expansion patterns that occurred during forward translation. They suggested that MST neurones had the appropriate structure to fulfil this role. These specialized 
detector networks could be thought of as templates designed to match a particular pattern of image motion. The Saito and Tanaka groups did not provide the specific details of the template construction but other heading models that followed used a similar design and provided more specific connectivity rules (Perrone 1987, 1990; Glünder 1990; Hatsopoulos and Warren 1991).

These original template models of human self-motion estimation could not process the inputs from combined translation and rotation self-motion. They were basically just expansion detectors (cf. Regan and Beverley 1978) and produce incorrect heading estimates when confronted with combined translation-rotation scenarios. Although they were biologically inspired and consistent with the known physiology, they could not compete with vector-based decomposition schemes that could solve the general self-motion problem. This deficit was overcome eventually when a model that could process translation and rotation was proposed (Perrone 1992). This model uses rotation detector networks to first detect the rotation visually. The output from these detectors is used to modify the heading templates to compensate for the rotation. The translation and rotation components of the flow field are never "separated out" locally as is the case in decomposition models. Since then, more elaborate template models which also incorporate eyemovement signals have been proposed (Beintema and van den Berg 1998).

A common general complaint against template models is that they require large numbers of detectors to handle the varieties of self-motion that can occur. We have, however, demonstrated that template models do not necessarily require large numbers of templates to operate successfully (Perrone and Stone 1994). In addition we have shown that the templates in our model share many of the properties of neurones in MST (Perrone and Stone 1998) and that heading can be directly encoded by individual MST neurones acting as templates. There is no need to postulate complex decomposition algorithms based on vector inputs.

In their analysis of visual interneurone properties in the fly (Krapp and Hengstenberg 1996; Dahmen et al., this volume) discovered a structure that is consistent with the basic template approach but they refer to the special detectors they found as "matched filters". They also propose that the function of the filters is to implement a form of vector decomposition. In this sense, their model does not fit cleanly into the category of template models discussed above. One way to evaluate models such as the Dahmen, Franz and Krapp model is to examine the types of inputs they require.

\section{Vector flow fields: The bane of self-motion estimation theorists}

The majority of current models of retino-centric instantaneous self-motion estimation assume that a velocity vector flow field is available to the navigating organism. While the notion of isolated points and velocity vectors is a useful 
theoretical construct for demonstrating that visual self-motion estimation is possible from retinal optic flow, it is far removed from the realities of everyday human visual navigation derived from retinal image motion. The two dimensional image motion generated from self-motion rarely arises from the movement of isolated points - instead surfaces and edges make up the major part of our visual environments and edges exist at a variety of orientations. A vector flow field consists of magnitude and direction measures at a number of locations in the visual field. I will examine both of these measures in detail in an attempt to convince the reader that vector flow fields are not realistic inputs to self-motion estimation in biological systems.

\subsection{Speed (vector magnitude)}

\section{Vector-based algorithms}

If an edge moves over a particular retinal location at $2 \%$, for instance, then vector-based algorithms require the value " 2 " to be passed onto the next stage of processing. If the image speed increases to $8 \%$ s then some coded value that is 4 times greater than the first speed must be sent to the self-motion processing unit. It requires a neurone that can provide a direct (e.g., rate coded) signal proportional to the speed. Evidence for such metrical coding of image speed has yet to be found in biological visual systems. For example, the most popular candidate for motion detection in insects, the Reichardt detector, produces an output that is influenced by the contrast and spatial structure of the input and which is not monotonically related to the image speed (Egelhaaf and Borst 1993). What we do find, however, is ample evidence for neurones in primate visual systems that are tuned to particular speeds.

Figure 1 shows re-plotted average speed tuning data from 109 MT cells collected by Maunsell and van Essen (1983). Similar data can be seen in a study by Lagae et al. (1993, their Fig. 9A). It is clear that at the level of MT there is no simple one-to-one relationship between the neurone's output and the speed of the edge moving across it. Some neurones do show low-pass or high-pass tuning in respect to image speed (see Lagae et al., their Fig. 9B) but these are in the minority and can hardly be described as exhibiting linear output properties. This means that in order to obtain the vector magnitude required for the processing of selfmotion, the decomposition models must assume the existence of a processing level above MT that uses some form of population code to derive the image speed. (e.g., Lappe et al. 1996).

The decomposition models cannot assume that neurones in cortical area MT of the primate brain, are producing the speed part of the velocity vectors they require in their computations. The MT neurones feed into area MST which has neurones with properties suitable for self-motion estimation (Perrone and Stone 1998). But the MST neurones appear to simply integrate the signals being gener- 
ated by the MT neurones (Tanaka and Saito 1989; Perrone and Stone 1998), without any intervening speed coding or "vector processing". Given that a signal proportional to the edge speed has yet to be found at any stage of the motion pathway, the decomposition models are faced with the problem of not having an obvious physiological correlate to the speed estimation part of their algorithms.

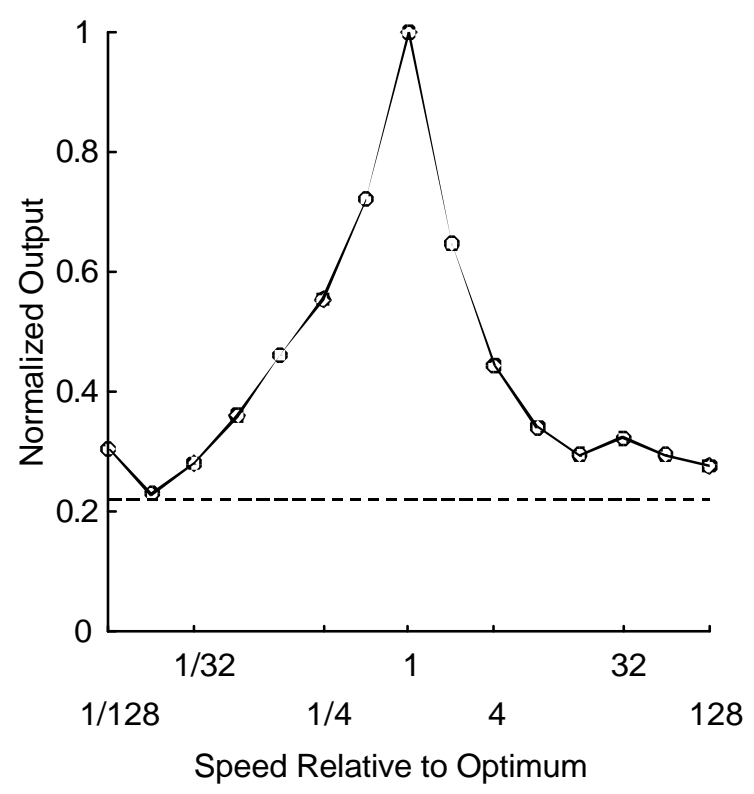

Fig. 1 Replotted mean speed tuning data from Maunsell and Van Essen (1983, their Fig. 6b). Horizontal dashed line corresponds to the average normalized background rate of firing of their sample.

\section{Template models. Speed tuning versus speed estimation}

How do template models avoid the "vector magnitude" problem outlined above? The simple answer is that these models make use of speed tuning, not speed estimation, and are built directly around the properties of MT neurones. Therefore the physiological counterpart to their input stage is clearly specified. The templates are based on the idea that if there is a good match between the velocity preference of the MT units making up the template and the image flow that is occurring on the retina, then a large signal will be generated in that template. If the match is not so good, the response will be less. The basic mechanism relies on speed and direction tuning as found in MT neurones (Maunsell and van Essen 1983; Albright 
1984). These neurones produce a large output when a feature moves across their receptive field at the correct speed and direction and less of an output when the velocity does not match, i.e., they have exactly the properties required by the template models (which is not surprising since the models evolved from them). For vector-based schemes the non-linear relationship between speed and neurone output is a problem (see above). Rather than being an integral part of their mechanism - as is the case for template models - the speed tuning is an obstacle that must be overcome by the vector-based models.

It should be pointed out, that template models of the type proposed by Perrone (1992) and Perrone and Stone (1994) cannot function properly with a single MT motion sensor at each location. Because it is not possible to anticipate the exact structure of the 3D environment, and hence the speed of the retinal image that will occur at a particular location, a number of different speed tunings must be included at each location. The range of speeds can be minimized by assuming particular environment layouts are more common than others (Perrone and Stone 1998; Dahmen et al., this volume) but usually more than one is required. A particular retinal image speed will activate one of the MT units at that location more than the others. Using a winner-takes-all scheme, the output of this MT neurone is passed onto the next stage. This is where the template models differ from the vector-based models. The speed preference (e.g., $2 \%$ ) of the winning MT neurone is not needed for later computations. It only becomes relevant when the structure of the environment needs to be determined (see Perrone and Stone 1994) and even then it is just the relative responses that are used, not the actual speeds.

The input to our template model is the neural activity from direction and speed tuned motion sensors (e.g., MT neurones). Examination of the figures in the early papers discussing the template model (Perrone 1992; Perrone and Stone 1994, 1998) may lead to some confusion in this regard. The inputs to the model are described and depicted as velocity vector fields. Because we did not have a model of MT neurones available that can be applied to image sequences, these template models have been implemented using velocity vectors as the initial input. The vectors are first passed through a stage involving idealized MT speed tuning curves and direction tuning curves to determine the simulated neurone output. We are currently attempting to bypass this unnecessary step by working with image sequences and models of MT neurones rather than idealized vector flow fields (Perrone 1997).

\subsection{Direction}

Obtaining the direction of the vector would seem straightforward for the visual system since there is ample evidence of neurones tuned for particular directions of motion (e.g., Middle Temporal neurones; Albright 1984). Nevertheless the problem is far from trivial. 
As was the case for speed estimation discussed above, there still needs to be a stage which converts the direction tuning into a measure of actual directions and the locus of this operation needs to be specified. In addition to this coding problem, there is an even bigger obstacle facing theorists who rely on knowledge of the velocity vector direction. For many self-motion scenarios, the image motion varies considerably from one retinal location to the next. This is because most scenes contain a range of objects at different distances from the moving observer and important information concerning the layout of the scene often occurs at nearby adjacent regions of the visual field. In order to register these small spatial variations, the analysis of the retinal image motion needs to be reasonably localized and invariably some of the 2D motion sensors performing the analysis will have receptive fields that contain a single moving edge. Once this occurs, the visual system is faced with the aperture problem (Wallach 1935) - motion in the direction parallel to the edge is invisible and only the component normal to the edge can be detected by the motion sensors. Figure 2 illustrates the problem and shows two flow fields, one where the aperture problem is assumed to not exist (a) and the other where it is present (b). The structure of the flow field is changed radically by the presence of the aperture problem.

\section{Vector-based approaches and the aperture problem}

The aperture problem has been largely ignored in the field of self-motion perception (see however, Perrone 1990, 1992; Heeger and Jepson; 1990). Many of the vector-based techniques rely on the detection of small vector differences that would be heavily masked by the large perturbations in speed and direction that arise from the aperture problem. Some self-motion estimation models claim resistance to the aperture problem because they incorporate spatial integration over large areas of the visual field (e.g., Perrone 1992; Heeger and Jepson 1992). This resistance however can only work up to a point and usually relies on certain assumptions about the distribution of edges in the scene. The analysis carried out by Dahmen et al is typical in that they simulate "noise" such as the aperture problem by perturbing the flow vector directions and speeds using a normal noise distribution that shifts the direction equally about the correct one. The noise is assumed to be statistically independent at neighbouring locations. Figure 2 demonstrates a weakness with this assumption because the direction perturbations can often be asymmetrically distributed and the image speeds are systematically distorted in certain areas of the visual field, not just increased or decreased randomly across the field. Humans routinely navigate safely in environments similar to that depicted in figure $2 b$, but most vector-based models of egomotion would have serious problems when faced with the input shown in this figure.

The aperture problem has mainly been addressed in the context of rigid twodimensional motion in the image plane. Techniques for overcoming the aperture problem under uniform 2D motion conditions (e.g., Adelson and Movshon 1982; 

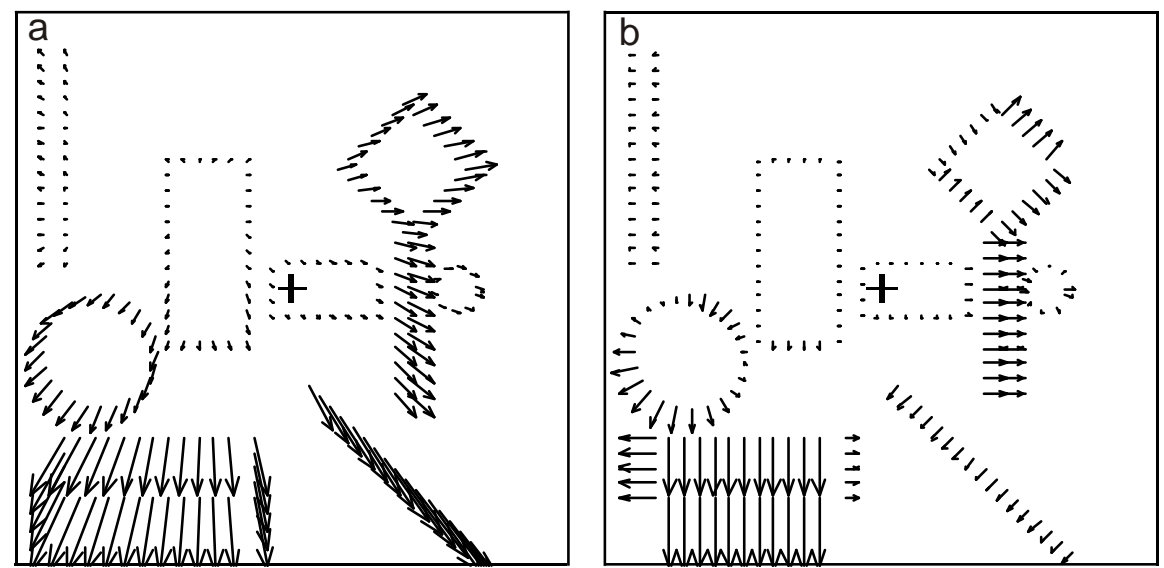

Fig. 2 Demonstration of the aperture problem. a This is the theoretical image motion that would be generated for an observer moving in a direction up and to the left of the viewing direction (marked with a cross). b Image motion for the same heading direction of the observer but it shows the result if only the motion normal to the edges in the scene could be detected.

Hildreth 1983; Yuille and Grzywacz 1988; Sereno 1993; Simoncelli and Heeger 1998) cannot be applied directly to the 3D self-motion situation. These techniques assume rigid 2D object motion and were not designed to accommodate the ambiguous speeds and directions endemic to 3D flow fields. You cannot "smooth" or average the vectors in figure $2 \mathrm{~b}$ without introducing large errors because in many cases adjacent vectors come from different objects separated in depth. More elaborate solutions to the aperture problem have been proposed which can deal with multiple objects, transparency and partial occlusion (e.g., Nowlan and Sejnowski 1995) although it is not clear how well such models can deal with the multi-speed, multi-direction motion inputs that result from observer motion. This model also requires a stage above MT in order to obtain the final image velocity from the activity of the "velocity units" computing the motion. It therefore suffers from the same problems outlined under speed tuning in Section 3.1.

\section{Template models and the aperture problem}

If one assumes that MT neurones are able to solve the aperture problem, then in theory the aperture problem should not cause template models any problems because MT neurones form the main input to these models. However, because the exact mechanisms for how MT neurones actually overcome the aperture problem are currently unknown, the template models cannot include this stage as part of their processing chain. We have begun exploring different possible methods for solving the aperture problem in the context of self-motion estimation and the 
template approach. Each heading template in our model of self-motion estimation is made up of a network that connects together particular sets of MT-like 2D motion sensors. For a particular preferred heading direction $(\alpha, \beta)$ coinciding with retinal image location $(\mathrm{x}, \mathrm{y})$, the direction preferences of the motion sensors are radially aligned around $(x, y)$. If $(\alpha, \beta)$ coincides with the actual heading direction, then the true image motion of an isolated point would be along one of these radial directions $(\phi)$. Assume that the speed of the point is $\mathrm{V} \rho / s$ ) and, for simplicity, that the template $2 \mathrm{D}$ motion sensor speed tuning matches this speed. If the point is replaced with an edge, then depending on its orientation, the motion of the edge normal could lie anywhere in a $180^{\circ}$ semi-circle centred around $\phi$. The speed of the edge will be reduced (relative to that of the point), by the cosine of the angle between the edge normal direction and $\phi$. This $180^{\circ}$ of possible directions could be sampled with a modest number of additional $2 \mathrm{D}$ motion sensors given the relatively broad directional tuning of MT units (Albright 1984). At each image location, the motion sensor tuned to $[\mathrm{V}, \phi]$ could be augmented by sensors tuned to $[0$, $\left.\pm 90^{\circ}\right],\left[\mathrm{V} \cos (60), \pm 60^{\circ}\right]$ and $\left[\mathrm{V} \cos (30), \pm 30^{\circ}\right]$.

One solution for template models is therefore to include an additional set of MT-like sensors into their detector networks which are tuned to a subset of the extra possible image motions that can arise as a result of the aperture problem. Initial tests of this concept using "artificial" inputs such as those in figure $2 b$ have been successful, but a thorough testing of the aperture problem solution cannot be undertaken until an image-based version of the model is implemented. Natural images contain features at a variety of spatial scales, contrasts and orientations and the motion "information" is much more complex than that depicted in figure $2 \mathrm{~b}$.

\section{Conclusion}

The vector flow field has become an integral part of many treatments of the visual self-motion estimation problem. Over the years researchers have either assumed its existence or sought after it as though it was some sort of Holy Grail - although I think a better analogy is that of a Siren luring sailors onto the rocks. The current physiological data better supports the view that primate visual systems do not use the vector flow field but instead have developed a solution around speed- and direction-tuned motion sensors. Template models have followed this lead and thus avoid many of the problems associated with vector flow field inputs. Even so, they still have a long way to go before they can provide an adequate description of biological self-motion estimation. The starting point for self-motion perception is the changing patterns of light falling onto the retinae of the eyes. Therefore the true input for self-motion estimation is a two-dimensional image sequence. Template models currently assume that MT neurone activity has been derived from the image motion. The challenge is to remove this assumption by simulating 
the MT stage as well. The next generation of self-motion models must be designed to work with natural image sequences and the vector flow field can be laid to rest.

\section{Acknowledgements}

Supported by NASA-Ames Grant NAG 2-1168

\section{References}

Adelson EH, Movshon JA (1982) Phenomenal coherence of moving visual patterns. Nature 300: 523-525

Albright TD (1984) Direction and orientation selectivity of neurons in visual area MT of the Macaque. J Neurophysiol 52: 1106-1130

Beintema JA, van den Berg AV (1998) Heading detection using motion templates and eye velocity gain fields. Vision Res 38: 2155-2179

Cutting JE (1986) Perception with an eye for motion. Bradford, Cambridge

Duffy CJ, Wurtz RH (1991a) Sensitivity of MST neurons to optic flow stimuli. I. A continuum of response selectivity to large-field stimuli. J Neurophysiol 65: 1329-1345

Duffy CJ, Wurtz RH (1991b) Sensitivity of MST neurons to optic flow stimuli. II. Mechanisms of response selectivity revealed by small-field stimuli. J Neurophysiol 65: 1346-1359

Dyre BP, Andersen GJ (1994) Statistical moments of retinal flow may be used to determine heading. Invest Ophthalmol Vis Sci 35: S 1269

Egelhaaf M, Borst A (1993) Movement detection in arthropods. In: Miles FA, Wallman J (eds) Visual motion and its role in the stabilization of gaze. Elsevier, Amsterdam, pp 53-77

Glünder H (1990) Correlative velocity estimation: Visual motion analysis, independent of object form, in arrays of velocity-tuned bilocal detectors. J Opt Soc Am A 7: 255-263

Gibson JJ (1950) The perception of the visual world. Houghton Mifflin, Boston

Hatsopoulos N, Warren WH (1991) Visual navigation with a neural network. Neural Networks 4: 303-317

Hausen K (1993) Decoding of retinal image flow in insects. In: Miles FA, Wallman J (eds) Visual motion and its role in the stabilization of gaze. Elsevier, Amsterdam, pp 203-235

Heeger DJ, Jepson AD (1992) Subspace methods for recovering rigid motion I: Algorithm and implementation. Int J Comp Vision 7: 95-177

Hengstenberg R (1982) Common visual response properties of giant vertical cells in the lobula plate of the blowfly Calliphora. J Comp Physiol A 149: 179-193

Hildreth EC (1983) The computation of the velocity field. Proc Roy Soc Lond B 221: 189-220

Hildreth EC (1992) Recovering heading for visually-guided navigation. Vision Res 32: 11771192

Koenderink JJ, van Doorn AJ (1975) Invariant properties of the motion parallax field due to the movement of rigid bodies relative to an observer. Opt Acta 22:773-791

Krapp HG, Hengstenberg R (1996) Estimation of self-motion by optic flow processing in single visual interneurons. Nature 384: 463-466

Lagae S, Raiguel S, Orban GA (1993) Speed and direction selectivity of macaque middle temporal neurons. J Neurophysiol 69: 19-39

Lappe M, Rauschecker JP (1993) A neural network for the processing of optic flow from egomotion in man and higher mammals. Neural Comput 5: 374-391

Lappe M, Bremmer F, Pekel M, Thiele A, Hoffmann KP (1996) Optic flow processing in monkey STS: A theoretical and experimental approach. J Neurosci 16: 6265-6285 
Longuet-Higgins HC, Prazdny K (1980) The interpretation of moving retinal images. Proc Roy Soc Lond B 208: 385-387

Maunsell JHR, van Essen DC (1983) Functional properties of neurons in the middle temporal visual area of the Macaque monkey. I. Selectivity for stimulus direction, speed, orientation. J Neurophysiol 49: 1127-1147

Nowlan SJ, Sejnowski TJ (1995) A selection model for motion processing in area MT of primates. J Neurosci 15: 1195-1214

Perrone JA (1987) Extracting 3-D egomotion information from a 2-D flow field: A biological solution? Opt Soc Am Tech Digest Series 22: 47

Perrone JA (1990) Simple technique for optical flow estimation. J Opt Soc Am A 7: 264-278

Perrone JA (1992) Model for the computation of self-motion in biological systems. J Opt Soc Am A 9: 177-194

Perrone JA (1997) Extracting observer heading and scene layout from image sequences. Invest Ophthalmol Vis Sci 38: S 481

Perrone JA, Stone LS (1994) A model of self-motion estimation within primate extrastriate visual cortex. Vision Res 34: 2917-2938

Perrone JA, Stone LS (1998) Emulating the visual receptive field properties of MST neurons with a template model of heading estimation. J Neurosci 18: 5958-5975

Regan D, Beverley KI (1978) Looming detectors in the human visual pathway. Vision Res 18 : 415-421

Rieger JH, Lawton DT (1985) Processing differential image motion. J Opt Soc Am A 2: 354-360

Rieger JH, Toet L (1985) Human visual navigation in the presence of 3D rotations. Biol Cybern 52: $377-381$

Royden CS (1997) Mathematical analysis of motion-opponent mechanisms used in the determination of heading and depth. J Opt Soc Am A 14: 2128-2143

Saito H, Yukie M, Tanaka K, Hikosaka K, Fukada Y, Iwai E (1986) Integration of direction signals of image motion in the superior temporal sulcus of the Macaque monkey. J Neurosci 6: $145-157$

Sereno ME (1993) Neural computation of pattern motion: modeling stages of motion analysis in the primate visual cortex. MIT Press, Cambridge, Mass

Simoncelli EP, Heeger DJ (1998) A model of neuronal responses in visual area MT. Vision Res 38: 743-761

Stone LS, Perrone JA (1997) Human heading estimation during visually simulated curvilinear motion. Vision Res 37: 573-590

Tanaka K, Hikosaka K, Saito H, Yukie, M, Fukada Y, Iwai E (1986) Analysis of local and widefield movements in the superior temporal visual areas of the macaque monkey. J Neurosci 6 : 134- 144

Tanaka K, Saito H (1989) Analysis of the motion of the visual field by direction, expansion/contraction, and rotation cells clustered in the dorsal part of the medial superior temporal area of the macaque monkey. J Neurophysiol 62: 626-641

Wallach H (1935) Über visuell wahrgenommene Bewegungsrichtung. Psychol Forsch 20: 325-380

Warren WH, Hannon DJ (1990) Eye movements and optical flow. J Opt Soc Am A 7: 160-168

Yuille AL, Gryzywacz NM (1988) A computational theory for the perception of coherent visual motion. Nature 333: 71-74

Zacharias GL, Caglayan AK, Sinacori JB (1985) A visual cueing model for terrain-following applications. J Guidance 8: 201-207 